\title{
Ultralow blocking temperature and breakdown of the giant spin model in $\mathrm{Er}^{3+}$-doped nanoparticles
}

\author{
W. Van den Heuvel, ${ }^{1}$ V. K. Tikhomirov, ${ }^{2}$ D. Kirilenko, ${ }^{3}$ N. Schildermans, ${ }^{2}$ L. F. Chibotaru, ${ }^{1}$ J. Vanacken, ${ }^{2}$ P. Gredin,${ }^{4}$ \\ M. Mortier, ${ }^{4}$ G. van Tendeloo, ${ }^{3}$ and V. V. Moshchalkov ${ }^{2}$ \\ ${ }^{1}$ Chemistry Department, Katholieke Universiteit Leuven, Leuven B-3001, Belgium \\ ${ }^{2}$ Institute for Nanoscale Physics and Chemistry (INPAC), Katholieke Universiteit Leuven, Leuven B-3001, Belgium \\ ${ }^{3}$ Physics Department, Antwerpen University, Antwerpen B-2020, Belgium \\ ${ }^{4}$ Laboratoire de Chimie de la Matière Condensée, Ecole Nationale Supérieure de Chimie de Paris, \\ UPMC-Paris 6, CNRS UMR 7574, F-75005, Paris France
}

(Received 23 March 2010; revised manuscript received 3 June 2010; published 13 September 2010)

\begin{abstract}
The magnetization of luminescent $\mathrm{Er}^{3+}$-doped $\mathrm{PbF}_{2}$ nanoparticles (formula $\mathrm{Er}_{0.3} \mathrm{~Pb}_{0.7} \mathrm{~F}_{2.3}$ ) has been studied. Despite the high concentration of the doping $\mathrm{Er}^{3+}$ ions and relatively large size $(8 \mathrm{~nm})$ of these nanoparticles we have found no deviation between field-cooled and zero-field-cooled magnetization curves down to $T$ $=0.35 \mathrm{~K}$, which points out an ultralow blocking temperature for the reversal of magnetization. We also have found strongly deviating magnetization curves $M(H / T)$ for different temperatures $T$. These results altogether show that the investigated nanoparticles are not superparamagnetic, but rather each $\mathrm{Er}^{3+}$ ion in these nanoparticles is found in a paramagnetic state down to very low temperatures, which implies the breakdown of the Néel-Brown giant spin model in the case of these nanoparticles. Calculations of magnetization within a paramagnetic model of noninteracting $\mathrm{Er}^{3+}$ ions completely support this conclusion. Due to the ultralow blocking temperature, these nanoparticles have a potential for magnetic field-induced nanoscale refrigeration with an option of their optical localization and temperature control.
\end{abstract}

DOI: 10.1103/PhysRevB.82.094421

PACS number(s): 75.75.-c, 75.10.Dg, 75.20.Ck

\section{INTRODUCTION}

The physics of magnetic nanoparticles is an active field of research for already a few decades due to new magnetic properties which arise at the nanoscale and to numerous applications. ${ }^{1}$ One of the central demands in these applications is the fabrication of nanoparticles with a narrow size distribution and their arrangement in two- and threedimensional lattices with controllable interparticle spacing, which was recently achieved by new synthetic approaches and self-assembly. ${ }^{2-5}$ For the storage applications, it is desirable also to have nanoparticles with large anisotropy energy while keeping their volume small. ${ }^{6}$ Magnetic nanoparticles have been attracting recently a substantial interest also for hyperthermal treatment of cancer cells, nuclear magnetic resonance imaging and nanolabeling, ${ }^{7,8}$ as well as for sitespecific drug delivery and manipulating cell membranes. ${ }^{9,10}$ A great potential for future applications of nanoparticles is related to magnetic field-induced refrigeration based on the magnetocaloric effect, which is considered as an alternative to a traditional vapor-cycle refrigeration. ${ }^{11-13}$ Here rare-earth intermetallic compounds have been considered as most promising for magnetic refrigeration due to the large magnetic moments in the ground state of their rare-earth components, such as Gd, Er, Ho, and Dy. ${ }^{11,13}$ Luminescent nanoparticles also have been proven themselves useful as optical nanolabels, ${ }^{7}$ nanoheaters, ${ }^{14}$ and nanosensors of magnetic field. ${ }^{15}$ Hence, an eventual combination of magnetic and luminescent properties in one nanoparticle would be of advantage.

In this paper we investigate the magnetization of luminescent $\mathrm{Er}^{3+}$-doped $\mathrm{PbF}_{2}$ nanoparticles, formula $\mathrm{Er}_{0.3} \mathrm{~Pb}_{0.7} \mathrm{~F}_{2.3}$. The nanoparticles were either embedded in bulk glass- ceramics host or extracted from this host to form freestanding nanopowder according to procedure, ${ }^{16}$ as described further in the Sec. II. We find that, in both cases, despite high concentration of the doping $\mathrm{Er}^{3+}$ ions and relatively large size $(8 \mathrm{~nm})$ of these nanoparticles, they show no blocking temperature down to $T=0.35 \mathrm{~K}$ as proved by field-cooled (FC) and zero-field-cooled (ZFC) magnetization experiments down to $T=0.35 \mathrm{~K}$. Moreover, the magnetization curves, when drawn as function of the ratio of applied magnetic field and temperature, $H / T$, strongly deviate from each other, resembling an anisotropic superparamagnetic behavior. ${ }^{1}$ These facts enforced us to conclude that the Néel-Brown model, ${ }^{17,18}$ based on the concept of giant (total) spin and successfully used for other nanoparticles, cannot be applied to the investigated $\mathrm{Er}^{3+}$-doped nanoparticles, which are therefore rather paramagnetic than superparamagnetic. We also performed simulations of measured magnetization and susceptibility within a model of noninteracting $\mathrm{Er}^{3+}$ ions which entirely supported this conclusion.

\section{EXPERIMENTAL}

First, the bulk oxyfluoride $\mathrm{Er}^{3+}$-doped nanoscaled glassceramics (NGC) 32( $\left.\mathrm{SiO}_{2}\right) 9\left(\mathrm{AlO}_{1.5}\right) 31.5\left(\mathrm{CdF}_{2}\right) 18.5\left(\mathrm{PbF}_{2}\right)$ $5.5\left(\mathrm{ZnF}_{2}\right): 3.5\left(\mathrm{ErF}_{3}\right)$, mol \%, has been prepared as described in Ref. 19. The $\mathrm{Er}^{3+}$ ions nucleate the growth of $\mathrm{PbF}_{2}$-based crystalline nanoparticles in this NGC on heat treatment and therefore up to $100 \%$ of $\mathrm{Er}^{3+}$ ions are incorporated into these nanoparticles (Refs. 14-16 and references therein). Further, the nanoparticles have been extracted from the NGC by means of chemical etching as described in Ref. 16.

The transmission electron microscope (TEM) images of the nanoparticles/nanopowder are presented in Figs. 

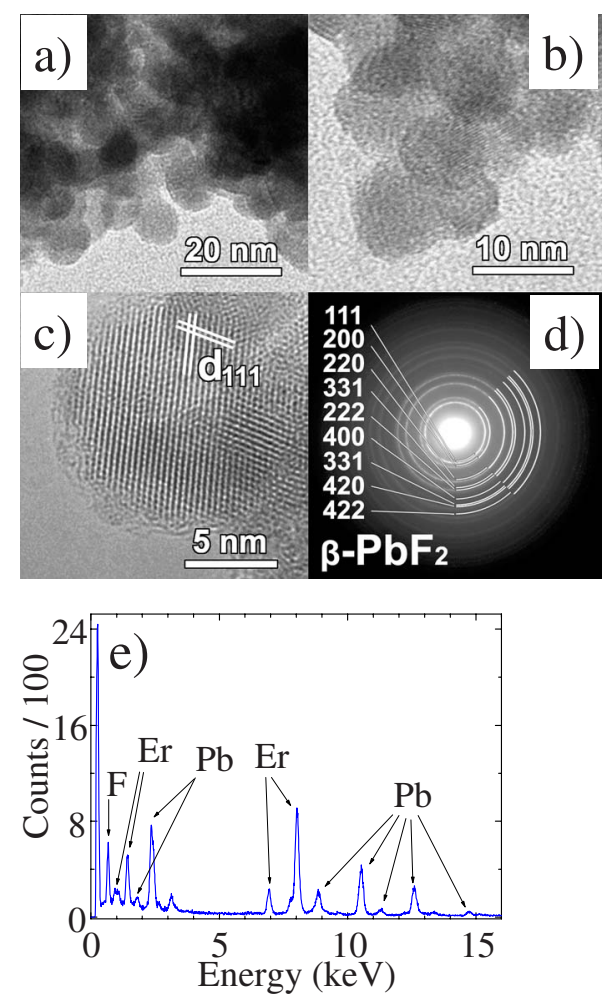

FIG. 1. (Color online) [(a) and (b)] TEM images of nanoparticles with different spatial resolution. Grey and black round balls of about $8 \mathrm{~nm}$ diameter are the nanoparticles comprising the respective aggregates/nanopowder. (c) TEM image of single nanoparticle, where crystalline planes $(1,1,1)$ of $\beta-\mathrm{PbF}_{2}$ are indicated as $d_{111}$. (d) Electron-diffraction pattern taken from nanopowder, where the diffraction rings from certain planes of the $\beta-\mathrm{PbF}_{2}$ are indicated by the straight lines and the respective Miller indexes. (e) TEM EDX spectra taken from the nanopowder; the observed peaks are labeled.

1(a)-1(c); they show that the nanoparticles are spherical and homogeneous in diameter, which is about $8 \mathrm{~nm}$. The electron-diffraction pattern and TEM energy-dispersion x-ray spectroscopy (TEM EDX) of the nanoparticles/nanopowder are shown in Figs. 1(d) and 1(e), respectively. The diffraction pattern in Fig. 1(d) indicates that the structure of the nanoparticles is a face-centered-cubic structure of the classic substituted fluorite [or $\beta-\mathrm{PbF}_{2}$ (Ref. 19)], which is known to be in case of $\mathrm{Er}^{3+}$ doping the $\mathrm{Er}_{x} \mathrm{~Pb}_{1-x} \mathrm{~F}_{2+x}$, where $x=0.29$, or approximately $\mathrm{Er}_{0.3} \mathrm{~Pb}_{0.7} \mathrm{~F}_{2.3}$ (Refs. 20 and 21 and references therein). The lattice parameter of the nanoparticles, Fig. 1(d), is slightly shorter than in $\beta-\mathrm{PbF}_{2}$ due to smaller ionic radii of $\mathrm{Er}^{3+}$ ions compared to $\mathrm{Pb}^{2+}$, as argued in Ref. 19.

When doing the TEM EDX experiments, Fig. 1(e), we have noted that the fluorine ions tend to move away from the area irradiated by the electron beam due to high superionic conductivity of the $\beta-\mathrm{PbF}_{2}$ (Ref. 21) and the negative charge of the electron beam. Therefore, the proportion of $\mathrm{Er}^{3+}$ and $\mathrm{Pb}^{2+}$ ions can be certainly found in TEM EDX experiments while the proportion of registered $\mathrm{F}^{-}$ions slightly depends on the parameters of the electron beam. As we have not found in EDX spectra any substantial admixtures of other ions apart from $\mathrm{Er}^{3+}, \mathrm{Pb}^{2+}$, and $\mathrm{F}^{-}$, [Fig. 1(e)], the proportion of $\mathrm{F}^{-}$ions was estimated exactly by charge compensation criterion, which corresponds to the classic substituted fluorite $\mathrm{Er}_{x} \mathrm{~Pb}_{1-x} \mathrm{~F}_{2+x}$, where $x=0.29$, or approximately to $\mathrm{Er}_{0.3} \mathrm{~Pb}_{0.7} \mathrm{~F}_{2.3}$.

The $\mathrm{Er}_{x} \mathrm{~Pb}_{1-x} \mathrm{~F}_{2+x}$ is known as a stoichiometric compound of tveitite type and rare-earth ions dissolve/disperse in this compound without clustering. The high luminescence yield in these nanoparticles ${ }^{22}$ proves high solubility/dispersion of the $\mathrm{Er}^{3+}$ dopants in tveitite structure because the clustering of the dopants could result in the concentration quenching of luminescence.

Both the diameter of the nanoparticles and concentration of $\mathrm{Er}^{3+}$ ions in these nanoparticles are intrinsically predefined by their preparation process, and therefore they cannot be varied, Refs. 14-16 and 19. The $\mathrm{Er}^{3+}$ ions nucleate the growth of the nanocrystalline phase in the nanoscaled glassceramics template, Ref. 19, the chemical composition of which was adjusted to obtain the nanocrystalline phase of stoichiometric composition $\mathrm{Er}_{0.3} \mathrm{~Pb}_{0.7} \mathrm{~F}_{2.3}$, otherwise the nanocrystalline phase did not precipitate. On the other hand, the diameter of $\mathrm{Er}_{0.3} \mathrm{~Pb}_{0.7} \mathrm{~F}_{2.3}$ nanoparticles was predefined by diffusion length of fluorine ions on the heat-treatmentinduced nanoceramic of the precursor glass, Ref. 16.

For the measurements of magnetization, a sample of the nanoparticles powder has been aggregated in a small cylinder pellet about $2 \mathrm{~mm}$ diameter and $2 \mathrm{~mm}$ height. The bulk NGC samples with a mass of about $40 \mathrm{mg}$ were chipped from the larger pieces of bulk NGC, which weighed up to 10-20 g. The magnetization has been measured with vibrating sample magnetometer (VSM), superconducting quantum interference device (SQUID), and Hall probe magnetometer in the temperature ranges $300-1.85 \mathrm{~K}, 15-3.5 \mathrm{~K}$, and $1.85-$ $0.35 \mathrm{~K}$, respectively, using liquid $\mathrm{He}^{4}$ and $\mathrm{He}^{3}$ cooling agents, as appropriate.

\section{RESULTS}

Figure 2 shows the temperature dependence of magnetization $M$ of the nanopowder obtained with (a) VSM, (b) SQUID, and (c) Hall probe techniques, respectively, in the $\mathrm{ZFC}$ and $\mathrm{FC}$ regimes. $\mathrm{Er}^{3+}$ is known as one of the most magnetic ions with the magnetic moment of $\sim 10 \mu_{\mathrm{B}}$ in the ground state $(J=15 / 2)$. These ions are responsible without doubts for the magnetic properties of the investigated nanoparticles. The deviation point in $\mathrm{ZFC}$ and $\mathrm{FC}$ temperature dependencies would indicate the blocking temperature $T_{\mathrm{b}}$, below which the magnetic moments of the sample get frozen, i.e., they cannot be thermally or magnetic field reoriented. ${ }^{23-25}$ However, the ZFC and FC curves were coincident in the whole temperature range, from 300 down to $0.35 \mathrm{~K}$, Figs. 2(a)-2(c), at any applied constant magnetic field $H$. This points out the absence of blocking temperature down to $0.35 \mathrm{~K}$, the lowest temperature which can be achieved with our $\mathrm{He}^{3}$ cryostat. This means that the magnetic moments of $\mathrm{Er}^{3+}$ in these nanoparticles still can be reoriented, either thermally or by applying an external magnetic field, even at very low temperatures. The qualitatively same result has been obtained for the bulk NGC samples with the magnitude of magnetization of about twice lower than in the nanopowder because in the NGC the nanoparticles comprise 

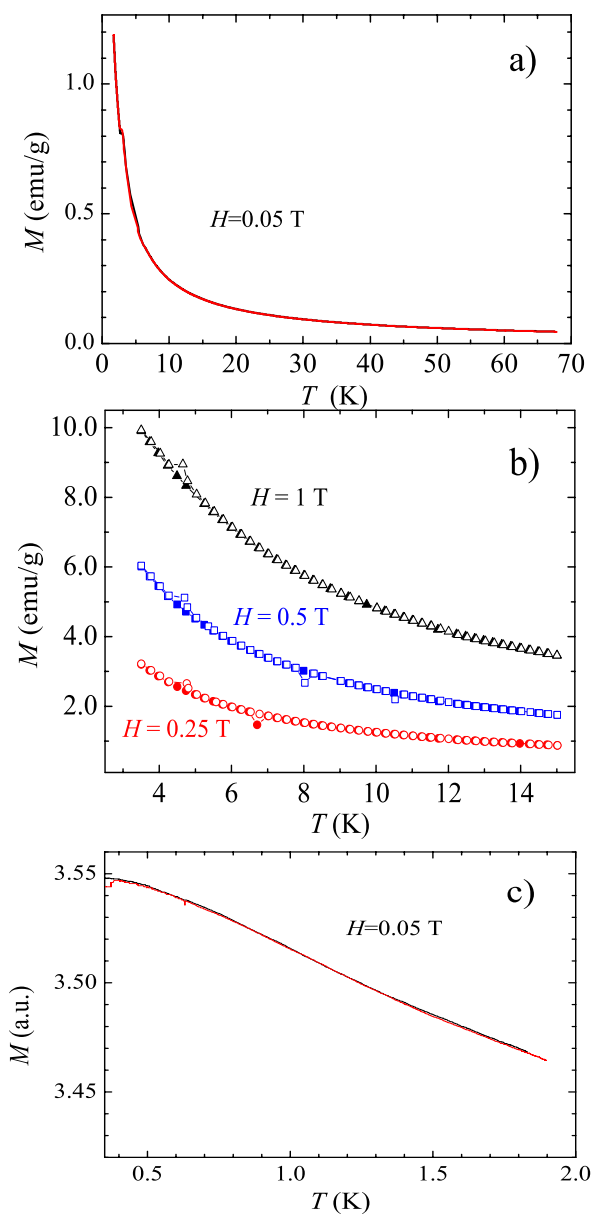

FIG. 2. (Color online) Temperature dependence of magnetization $M$ in $\mathrm{Er}_{0.3} \mathrm{~Pb}_{0.7} \mathrm{~F}_{2.3}$ nanopowder at indicated external magnetic fields. Measurements have been done using the (a) VSM, (b) SQUID, (c) and Hall probe setups. The dependences for ZFC and FC regimes were coincident at all applied external fields $H$.

only about half of the total mass of the sample. ${ }^{16,22}$

To attempt an attribution of the magnetic behavior shown in Fig. 2 to a known type of magnetism we have plotted in Fig. 3 (solid black line) the experimental temperature dependence of $1 / \chi$, where $\chi$ is the low-field $(H=0.05 \mathrm{~T})$ Van Vleck magnetic susceptibility $(\chi=M / H)$. Figure 3 shows that $1 / \chi(T)$ gradually approaches the origin of coordinates, as it follows from the extrapolation of the low-temperature part of the curve from the lowest measured point at $1.85 \mathrm{~K}$. The same result has been obtained for the bulk NGC samples and therefore is not shown here. On the basis of this behavior of the inverse susceptibility we conclude that it cannot be attributed to any type of magnetic ordering in the investigated temperature domain. Indeed, contrary to what we observe here, $1 / \chi(T)$ in ferro and ferri ordered bulk magnets has an intercept with the horizontal $(T)$ axis while in antiferromagnets and spin glasses it intersects the vertical $(1 / \chi)$ axis. ${ }^{26}$ In the case of nanoparticles, for temperatures lower than Curie/Néel temperature $T_{\mathrm{c}}$ of bulk material, the magnetic moments of metal ions order into a single magnetic domain which reorients freely as a single magnetic moment (giant spin). ${ }^{17}$ Due to the shape and magnetocrystalline anisotropy of nanoparticles the free rotations of their giant

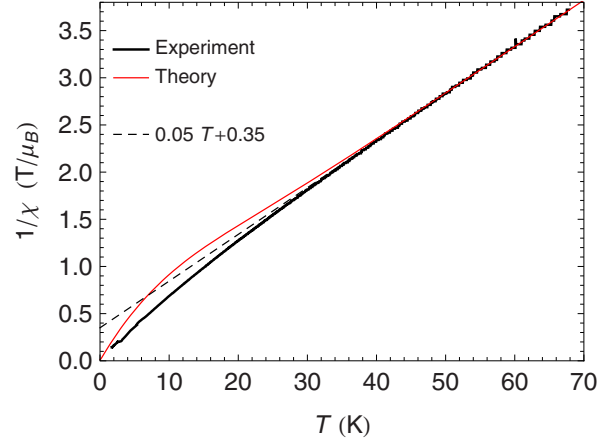

FIG. 3. (Color online) Experimental (solid black curve) and theoretical (thin curve) temperature dependences of the inverse magnetic susceptibility in $\mathrm{Er}_{0.3} \mathrm{~Pb}_{0.7} \mathrm{~F}_{2.3}$ nanopowder normalized per $\mathrm{Er}^{3+}$ ion and determined from the magnetization at $0.05 \mathrm{~T}$. The extrapolation of high-temperature linear part $(T>40 \mathrm{~K})$ of these dependences down to the low-temperature region is shown by dashed line. The experimental dependence $1 / \chi$ has been divided by a factor of 2.0, as discussed in the text. Crystal-field parameters for the theoretical curve were taken at $x=-0.375$ and $W=0.959 \mathrm{~cm}^{-1}$.

spins become progressively hindered so that for temperatures lower than the blocking temperature $T_{\mathrm{b}}$ [more exactly $T_{\mathrm{b}}$, the point of deviation of $M_{\mathrm{FC}}$ from $M_{\mathrm{ZFC}}$ (Ref. 24)] the susceptibility does not follow anymore the Curie-Weiss law. Instead $M_{\mathrm{FC}} / H$ tends to saturation while $M_{\mathrm{ZFC}} / H$ (the initial susceptibility) to zero, after passing through a maximum. ${ }^{1}$ The blocking temperature of typical nanoparticles amounts to several tens of kelvin [for instance, $T_{\mathrm{b}}=37.5 \mathrm{~K}$ in $\mathrm{CoPt}_{3}$ nanoparticles with $D=6 \mathrm{~nm}$ (Ref. 27)] and increases quickly with their diameter. The corresponding $1 / \chi(T)$ curves will bend upward at $T_{\mathrm{b}}$ for both kinds of magnetization, ${ }^{28}$ a behavior not observed in Fig. 3 within the measured temperature domain.

For $T>T_{\mathrm{b}}$ the nanoparticles show a superparamagnetic behavior ${ }^{1}$ with magnetic susceptibility following the CurieWeiss law: ${ }^{1,26}$

$$
\chi(T)=\frac{C}{T-\theta}
$$

with the Curie-Weiss parameter depending on interparticle interaction $\left(J_{\text {inter }}\right)$ and the giant spin of the nanoparticles $\left(S_{\mathrm{n}}\right)$ as $\theta \sim J_{\text {inter }} S_{\mathrm{n}}\left(S_{\mathrm{n}}+1\right) . J_{\text {inter }}$ includes contributions from exchange and dipolar interactions and is very small for distances $\sim 10 \mathrm{~nm}$. However, because $S_{\mathrm{n}}$ amounts to several thousands for nanoparticles with diameters of several nanometers $\left(S_{\mathrm{n}}=m / g \mu_{\mathrm{B}}\right.$, where $m$ is the magnetic moment and $g \approx 2$ ), $\theta$ can reach few tens of kelvin. ${ }^{1,24}$ Actually, because $\theta<T_{\mathrm{b}}$ the superparamagnetic behavior of nanoparticles in Eq. (1) is observed not for $T>\theta$ but for $T>T_{\mathrm{b}}$. In our case the Curie-Weiss parameter is also non-negligible (extrapolation of the dashed curve in Fig. 3 gives the intercept $\theta=$ $-7 \mathrm{~K})$. However, we cannot interpret the obtained negative Curie-Weiss parameter as an indicator of antiferromagnetic ordering of giant spins of the nanoparticles at lower temperatures. Indeed, if this would be the case the inverse susceptibility function will deviate upward from a straight line with lowering the temperature. Contrary to this, Fig. 3 shows an 

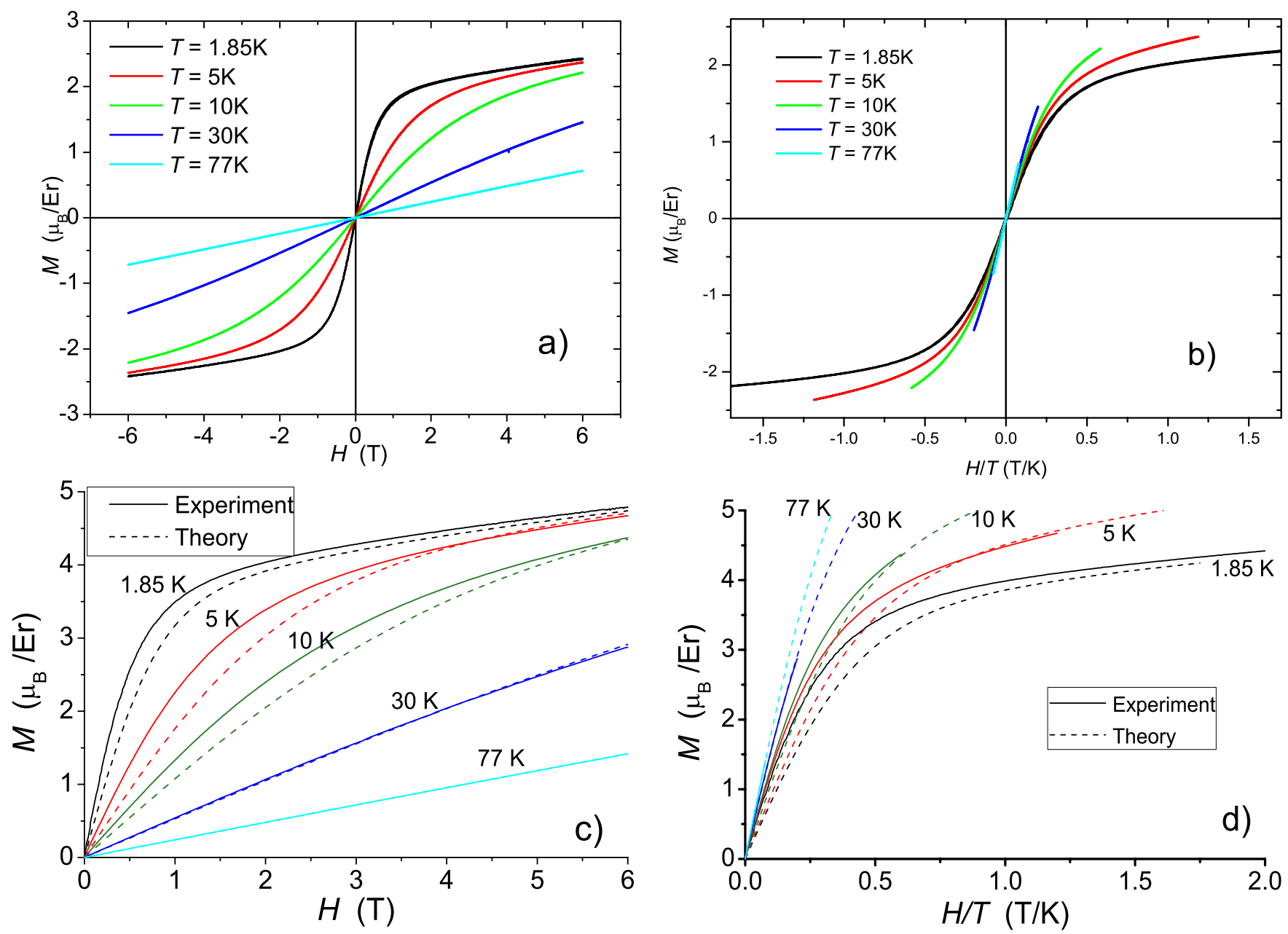

FIG. 4. (Color online) [(a) and (b)] The experimental field dependence of the magnetization in $\mathrm{Er}_{0.3} \mathrm{~Pb}_{0.7} \mathrm{~F}_{2.3}$ nanopowder, normalized per $\mathrm{Er}^{3+}$ ion. [(c) and (d)] The theoretical and experimental field dependences of the magnetization, while the experimental dependences have been multiplied by factor of 2.0, as in Fig. 3, for comparison with the theoretical data. Crystal-field parameters are the same as in Fig. 3.

opposite behavior, $1 / \chi(T)$ bends downward from the straight line starting from $T \approx 40 \mathrm{~K}$ and tends to zero down to very low temperatures. We may conclude therefore that the observed behavior of the inverse susceptibility cannot be accounted for by interaction and blocking of giant spins of the nanoparticles. This actually means that the susceptibility measurements evidence against the existence of giant spins (ordered magnetization domains) in our nanoparticles within the whole investigated temperature range-otherwise they would manifest themselves at low temperatures as in conventional magnetic nanoparticles.

Figure 4 shows the experimental magnetization curves (a) $M$ vs $H$ and (b) $M$ vs $H / T$ of the nanopowder at the indicated temperatures. Similar results have been obtained for the bulk NGC, again with about twice lower values due to the magnetic nonactive glass host component embedding the $\mathrm{Er}_{0.3} \mathrm{~Pb}_{0.7} \mathrm{~F}_{2.3}$ nanocrystals. For both nanopowder and bulk NGC materials, the $M(H)$ curves show no hysteresis, i.e., the coercive field is zero, and the magnetization does not reach saturation, even for fields up to $10 \mathrm{~T}$ (not shown here).

The curves in Fig. 4(a) resemble the behavior of superparamagnetic nanoparticles for $T>T_{\mathrm{b}}{ }^{23-25}$ The same curves drawn as function of the ratio $H / T$ [Fig. 4(b)] do not super- impose on each other pointing out an important contribution of magnetic anisotropy. Indeed, in the absence of this contribution the magnetization is described either by Brillouin or Langevin function, ${ }^{26}$ both depending on $H / T$; therefore, the functions $M(H / T)$ considered for different fixed values of $T$ would lie on the same curve. The behavior of magnetization similar to the one shown in Fig. 4(b) was also observed in other nanoparticles, e.g., in $\mathrm{Fe}_{1-x} \mathrm{Hg}_{x}$ (Ref. 28) and $\mathrm{CoPt}_{3},{ }^{27}$ and was called anisotropic superparamagnetism. ${ }^{1,27}$ In those nanoparticles the theoretical magnetization curves can be brought into accord with experiment by taking into account the anisotropy energy $E_{\mathrm{A}}$ of the total magnetization of the nanoparticle. ${ }^{27}$ However, this scenario cannot be applied to the nanoparticles investigated here for the following reasons. As already mentioned, in conventional nanoparticles the magnetization is well described within the Néel-Brown giant spin model. ${ }^{17,18}$ Within this model, the anisotropy energy (the difference of the energy of the giant spin aligned along and perpendicular to the easy axis of the nanoparticle) is equalized to the energy of the barrier for the reversal of magnetization of the nanoparticle, $E_{\mathrm{B}} \cdot{ }^{17}$ Now, since the blocking temperature for reversal of magnetization $T_{\mathrm{b}}$ is proportional to $E_{\mathrm{B}}$ (and $E_{\mathrm{A}}$ ), large deviations of magnetization 
curves from the predictions of the free magnetic moment model (described by the Langevin function) should be accompanied by large values of $T_{\mathrm{B}}$. This is indeed the case of conventional nanoparticles, e.g., of $\mathrm{CoPt}_{3}$ with $D=6 \mathrm{~nm}$, where $T_{\mathrm{B}}=37.5 \mathrm{~K}$ and deviations of magnetization from the Langevin function were observed up to $T=150 \mathrm{~K} .{ }^{27}$ By contrast, in our case the deviations of magnetization from the predictions of free magnetic moment model are of the same extent while the blocking temperature is practically zero. Thus the physics underlying the observed magnetization curves [Fig. 4(b)] is totally different.

The above analysis shows that both $\chi(T)$ and $M(H)$ of nanoparticles studied here cannot be described by the giant spin model for the whole considered temperature domain, down to $0.35 \mathrm{~K}$. This means that the magnetism of the $\mathrm{Er}^{3+}$-doped nanoparticles should be better described by a collection of paramagnetic moments of $\mathrm{Er}^{3+}$ ions, which reorient independently from each other down to very low temperatures. In other words, the nanoparticles are found in paramagnetic rather than superparamagnetic state. The ionic anisotropy on erbium sites, which is generally very strong in lanthanides, will not block the free reorientation of their magnetic moment. This blockage can only arise after taking into account their interaction. However, the dipolar and exchange interaction between distant $\mathrm{Er}^{3+}$ ions is negligible compared to the magnetic interaction between conventional nanoparticles (described by the giant spin) because the magnetic moments of individual metal ions are several orders of magnitude smaller than the total magnetic moments of the nanoparticles. Therefore the relevant magnetic interactions which lead to magnetic ordering in our case are the exchange interactions between neighbor $\mathrm{Er}^{3+}$ ions. The onset of magnetic ordering can be approximately inferred from the inflection point of $M(T)$ in Fig. 2(c), which gives the estimation $T_{\mathrm{c}} \approx 1 \mathrm{~K}$, and this is also the order of magnitude of the expected exchange contribution to $\theta$. The main contribution, however, comes from the anisotropic magnetism of individual $\mathrm{Er}^{3+}$ ions. Hence the observed magnetic properties of $\mathrm{Er}^{3+}$-doped nanoparticles for $T>1 \mathrm{~K}$ can be accounted for within a model of noninteracting erbium ions. We prove this in the next section by considering an idealized crystal-field model for the $\mathrm{Er}^{3+}$ ions.

\section{MODEL AND SIMULATIONS}

We show here that the Curie-Weiss behavior in Eq. (1) of susceptibility at $T>40 \mathrm{~K}$, the bending down of $1 / \chi(T)$ from a straight line at lower temperature and its approach to zero when $T \rightarrow 0$ K (Fig. 3) can be described by the effects of crystal-field splitting of the ${ }^{4} I_{15 / 2}$ ground-state multiplet of individual $\mathrm{Er}^{3+}$ ions. ${ }^{29}$ Crystal-field splitting in lanthanides is usually on the order of magnitude of hundreds of kelvin, comparable with the temperature scale of the measurements. Therefore, thermal population or depopulation of the crystalfield levels determines the temperature dependence of magnetization of the corresponding ions. The variation in the slope of inverse susceptibility curve is associated with the transition from a state where only the ground Kramers doublet is occupied to a state where both the ground and the first excited Kramers doublet(s) are occupied. This is accompanied by an increase in the average magnetic moment, as indicated by the decreasing slope (i.e., negative curvature) of $1 / \chi$ with increasing temperature. We see the same effect in the magnetization versus $H / T$ plots in Fig. 4(b). Perfectly coinciding curves are expected for the magnetization of a single Kramers doublet (Brillouin curves). Instead, the magnetization clearly increases with increasing temperature. Finally, the effect of excited Kramers doublets is also seen in the $M(H)$ plot at $1.85 \mathrm{~K}$ [Fig. 4(a)] as an almost linear increase in magnetization with the field after $1.5 \mathrm{~T}$ without signs of saturation up to $6 \mathrm{~T}$. This behavior results from Zeeman admixture of excited Kramers doublets to the ground state.

\section{A. Crystal-field calculations}

As it is seen in Fig. 1, the structure of the nanoparticles is face-centered cubic with a lattice parameter corresponding to the $\beta-\mathrm{PbF}_{2}$ phase. ${ }^{19}$ Therefore, to calculate the magnetic properties we consider the $\mathrm{Er}^{3+}$ ion in a cubic crystal field created by eight $\mathrm{F}^{-}$ions positioned at the vertices of a cube centered on $\mathrm{Er}^{3+}$. Actually this approximation is expected to be rather crude for two reasons. First, local charge compensation by $\mathrm{F}^{-}$or other anions can distort the cubic coordination environment considerably. Second, the site symmetry can be considered cubic only in the bulk of the $\mathrm{PbF}_{2}$ crystal. The nanoparticles have such a small size, however, that a non-negligible proportion of atoms are located near the surface of the particle. For example, if we assume that atoms belong to the "surface volume" if they are within $1 \mathrm{~nm}$ of the surface, we find that, in a spherical nanoparticle of radius 5 $\mathrm{nm}$, as much as $49 \%$ of the atoms is contained in the surface volume (assuming that the number of atoms per volume is constant over the nanoparticle). $\mathrm{Er}^{3+}$ ions near the surface will experience a deviation from the bulk cubic field. The strength and form of this perturbation are not known and we carry the calculation through in the assumption of perfect and uniform cubic crystal field. This would be enough for our purposes since we are only concerned with reproducing the basic features of the magnetism of $\mathrm{Er}^{3+}$-doped nanoparticles, which are expected to be weakly dependent on the details of the crystal field.

The calculations are based on the atomic ${ }^{4} I_{15 / 2}$ level only without consideration of higher atomic multiplets. The resulting Stark levels are characterized by irreducible representations $\Gamma_{7}+3 \Gamma_{8}+\Gamma_{6}$ of the cubic group. ${ }^{30}$ The cubic field is described by two parameters $x$ and $W$, defined in Refs. 31 and 32. The parameter $x$ determines the relative contributions of the fourth- and sixth-order angular-momentum operators to the crystal-field Hamiltonian while the parameter $W$ determines the energy scale of the crystal-field interaction. In Ref. 33 , the following values were determined for $\mathrm{Er}^{3+}$ centers in a $\mathrm{PbF}_{2}$ crystal with nonlocal charge compensation: $x=-0.375$ and $W=1.49 \mathrm{~cm}^{-1}$. We take these as starting values, keeping in mind that they may be adjusted to fit the experimental results. These parameters place the $\Gamma_{7}$ as ground state and an accidentally degenerate $\Gamma_{6}, \Gamma_{8}$ pair at $80 \mathrm{~cm}^{-1}$ higher. ${ }^{31}$ The Landé $g$ factor for the ground atomic multiplet $J=15 / 2$ equals to $6 / 5 .^{32}$ 


\section{B. Simulation of magnetic properties}

We have modeled the experimental magnetization curves of Fig. 4 by applying the field along the main symmetry axes, such as $C_{4}$ and $C_{3}$, and found that the theoretical curves for these directions deviate less than $5 \%$ from each other for fields lower than 6 T. Since the nanoparticles are randomly oriented with respect to the direction of the applied magnetic field, an average among the different crystalline orientations has to be taken into account but the small difference in magnetization along different axes makes such an averaging actually unnecessary. The experimental magnetization and susceptibility, recalculated per one $\mathrm{Er}^{3+}$ from the sample mass and the formula $\mathrm{Er}_{0.3} \mathrm{~Pb}_{0.7} \mathrm{~F}_{2.3}$, were found too large compared to the theoretical predictions for one erbium ion. Therefore, in order to obtain a quantitative correspondence with the experiment, Fig. 4, it was necessary to rescale the experimental curves. There are two experimentally accessible properties independent of the crystal-field parameters from which the scaling factor can be derived. The first is the slope of $1 / \chi$ in the high-temperature limit. It is crystal-fieldindependent because the splitting of the energy levels is irrelevant at temperatures higher than this splitting. The second is the intercept of $M(H)$ with the vertical $(H)$ axis in the limit $T \rightarrow 0$. This intercept equals the magnetic moment of the $\Gamma_{7}$ ground doublet. This state is independent from the parameters of cubic crystal field because $\Gamma_{7}$ occurs only once in the reduction $J=15 / 2 \rightarrow \Gamma_{6}+\Gamma_{7}+3 \Gamma_{8}$. The $\Gamma_{7}$ wave function is thus completely defined by symmetry; its magnetic moment is $m\left(\Gamma_{7}\right)=3.4 \mu_{\mathrm{B}}{ }^{32}$ Of course this is only valid insofar as the symmetry is exactly cubic, which is certainly not the case in our nanoparticles as discussed above. In the present case a reliable intercept cannot be derived from the $1.85 \mathrm{~K}$ curve, as there is some curvature in the higher field region [Fig. 4(c)]. Still lower temperatures would be needed for this. Moreover the limit $T \rightarrow 0$ cannot be attained in the paramagnetic regime due to the expected onset of magnetic ordering at $T \approx 1 \mathrm{~K}$ as mentioned in the end of Sec. III. We will therefore derive the scaling factor by using the susceptibility in the high-temperature domain. In the limit of very high temperature, exceeding the crystal-field splitting of the atomic multiplet ${ }^{4} I_{15 / 2}$, the slope of $1 / \chi$ is given by the Curie formula for ground-state angular momentum $J=15 / 2$ of a free $\mathrm{Er}^{3+}$ ion,

$$
\frac{1}{\chi T}=\frac{3 k}{\mu_{\mathrm{B}} g^{2} J(J+1)}=0.0487,
$$

where $\chi$ is in units of $\mu_{\mathrm{B}} / \mathrm{T}$. The calculations show that this slope actually remains constant in a broad domain, for temperatures down to several tens of kelvin. The measured $1 / \chi$ approaches linearity from about $45 \mathrm{~K}$, Fig. 3, with a slope of $\approx 0.099 \mathrm{~T} /\left(\mu_{\mathrm{B}} \mathrm{K}\right)$. Together with Eq. (2), this yields a scaling factor of $0.099 / 0.0487 \approx 2.0$. We can attribute this discrepancy to errors in the experimental estimation of erbium content and to possible quenching of magnetic moments on erbium ions due to their clustering which was observed in other erbium-doped materials. ${ }^{34,35}$

With the slope fixed by the scaling factor, the $\theta$ in hightemperature part of theoretical curve $1 / \chi(T)$ is made to co- incide with the experimental one by adjusting the crystalfield parameters. The $x$ parameter was kept constant at -0.375 while the $W$ parameter was lowered to $0.959 \mathrm{~cm}^{-1}$. In principle, the $x$ parameter could be adjusted to further refine the correspondence. We found, however, no improvement doing this and $x$ was kept to the value reported in Ref. 33 . Note that in all cases the model yields the negative sign of the Curie-Weiss parameter in Eq. (1), which shows that the simplified cubic crystal-field model reproduces correctly the increase in the magnetic moment on $\mathrm{Er}^{3+}$ sites with temperature.

Figures 4(c) and 4(d) show the theoretical magnetization curves for a single $\mathrm{Er}^{3+}$ ion within the above model, compared with the experimental measurements on the nanoparticles. The magnetization curves in these figures were calculated for field parallel with a cubic $C_{4}$ axis. Note that while the scaling factor and the $W$ parameter were determined from the susceptibility measurement alone, the agreement with the magnetization curves is satisfactory as well, and can be seen as an independent confirmation of the model.

There are still discrepancies between theory and experiment in the low-temperature region that could not be eliminated within the cubic crystal-field model applied here. This is not unexpected in view of the reservations we made concerning the validity of the cubic crystal-field approximation for our nanoparticles. The large reduction in the $W$ parameter, from $1.49 \mathrm{~cm}^{-1}$ in Ref. 33 to $0.995 \mathrm{~cm}^{-1}$ here, should probably also be regarded as an indication of the deviation from uniform, cubic potential that the $\mathrm{Er}^{3+}$ ions experience in the nanoparticles. We note that the actual crystal field for $\mathrm{Er}^{3+}$ ions is basically unknown. The erbium sites feel different crystal field at different locations in the nanoparticle while the structure of each $\mathrm{Er}^{3+}$ environment cannot be determined from the TEM (Fig. 1). Besides it is influenced by the presence of randomly distributed $\mathrm{F}^{-}$ions. On the other hand the calculations show that varying the parameters of a noncubic crystal field it is possible to reproduce well the experimental curve $1 / \chi(T)$ in Fig. 3.

\section{DISCUSSION AND CONCLUSIONS}

We have studied the magnetic properties of luminescent $\mathrm{Er}^{3+}$-doped $\mathrm{PbF}_{2}$ nanoparticles when embedded in glassceramics host and when extracted from this host. Our main finding is the ultralow value of blocking temperature of reversal of magnetization in these nanoparticles, for which the upper limit was estimated to be at $0.35 \mathrm{~K}$. This is the more surprising that the nanoparticles have relatively large diameters and high concentration of erbium ions. In combination with other observed magnetic properties, Figs. 3 and 4(b), this result implies that the investigated nanoparticles are not superparamagnetic but simply paramagnetic, i.e., described by independent dynamics of individual magnetic moments of $\mathrm{Er}^{3+}$ ions. This means the breakdown of the giant spin model in application to $\mathrm{Er}^{3+}$-doped nanoparticles investigated here.

One of the consequences of the independent-localmoments model is that the shape anisotropy of the nanoparticle, which is the main source of magnetic anisotropy in conventional nanoparticles, ${ }^{17}$ does not play a role here. By 
contrast in the present model the main contribution to the deviation of magnetization curves from conventional (spin free) ones is due to a combination of strong spin-orbit coupling and the crystal-field splitting on the $\mathrm{Er}^{3+}$ sites. We note that the observed effects are not related directly to the local (ionic) anisotropy on the erbium sites but only to the presence of low-lying crystal-field levels with strongly differing magnetic moments. For instance, in the simplified crystalfield model considered here the local magnetic moments on the $\mathrm{Er}^{3+}$ sites are perfectly isotropic (no easy axes of magnetization) due to the assumed cubic symmetry of the $\mathrm{Er}^{3+}$ environment. Note that the independent-local-moments model is fully consistent with the inferred very low value of $T_{\mathrm{b}}$ because there is no blockage of magnetization in a system of noninteracting magnetic ions.

The proposed paramagnetic interpretation of measured magnetic properties has been supported by simulations done within the model of magnetically noninteracting $\mathrm{Er}^{3+}$ ions with a further simplification that the erbium ions resided in perfectly cubic sites. Despite the fact that such a crystal-field model is not supported by recent luminescence experiments for the investigated nanoparticles, ${ }^{19,29}$ it allows to reproduce all qualitative features of magnetization and susceptibility (Figs. 3 and 4). In order to perform more realistic simulations the determination of the actual structure of the nearest environment of $\mathrm{Er}^{3+}$ ions in the investigated nanoparticles is indispensable. This task can be accomplished by using neutron-scattering techniques. Detailed knowledge of crystal field on $\mathrm{Er}^{3+}$ sites will also allow to get more insight into the mechanism of extraordinary suppression of green luminescence of these nanoparticles in strong magnetic field. ${ }^{36}$

Interestingly, lanthanides doped in different metals have shown pretty high blocking temperatures at much lower concentrations. ${ }^{37}$ For instance, a single crystal of $\mathrm{Y}$ doped with only $5 \%$ of erbium ions shows $T_{\mathrm{b}}=5.6 \mathrm{~K} .{ }^{37}$ The large difference in the value of blocking temperature in the present case is probably explained by the fact that $\mathrm{Er}^{3+}$ are doped into the insulating $\mathrm{PbF}_{2}$ host which, in contrast to the metals, does not transmit efficiently the exchange interactions between lanthanide ions. Also lanthanides in insulating bulk materials exhibit very small exchange interaction, which is manifested in the absence of magnetic order till quite low temperatures even in concentrated systems. ${ }^{38}$ Moreover, the inverse susceptibility in $\mathrm{Yb}_{2}\left(\mathrm{SO}_{4}\right)_{3} \cdot 8 \mathrm{H}_{2} \mathrm{O}$ (Ref. 38) was found to follow a similar temperature behavior as in Fig. 3 which was explained by similar reasons as in the present work. ${ }^{39}$ We expect therefore that magnetic properties similar to $\mathrm{Er}^{3+}$-doped nanoparticles will be observed also in $\mathrm{Er}^{+}$-doped bulk insulators.

An implication of our findings is that the earlier known transparent and luminescent $\mathrm{Er}^{3+}$-doped $\mathrm{PbF}_{2}$ nanoparticles ${ }^{16}$ may also be used for nanoscale refrigeration, in particular, at temperatures below $1 \mathrm{~K}$, due to high magnetic moment in the ground state of the $\mathrm{Er}^{3+}$ and the absence of the blocking temperature down to at least $0.35 \mathrm{~K}$. Besides, with these nanopowder/nanoparticles the refrigerated nanovolume and its temperature can be detected distantly by means of their luminescence properties. This is an advantage compared to other magnetic refrigeration materials, which are neither nanoscaled nor luminescent.

\section{ACKNOWLEDGMENTS}

We gratefully acknowledge support from Katholieke Universiteit Leuven, Belgium, under Grants No. EF/05/005 and No. GOA/09/005 and project Methusalem 08/05 founded by the Flemish government. W. V. d. H. acknowledges support from the Research Foundation-Flanders (FWO).
${ }^{1}$ S. Bedanta and W. Kleemann, J. Phys. D 42, 013001 (2009).

${ }^{2}$ S. Sun, C. B. Murray, D. Weller, L. Folks, and A. Moser, Science 287, 1989 (2000).

${ }^{3}$ V. F. Puntes, K. M. Krishnan, and A. P. Alivisatos, Science 291, 2115 (2001).

${ }^{4}$ E. V. Shevchenko, D. V. Talapin, A. Kronowski, F. Wiekhorst, J. Kötzler, M. Haase, A. L. Rogach, and H. Weller, Adv. Mater. (Weinheim, Ger.) 14, 287 (2002).

${ }^{5}$ E. V. Shevchenko, D. V. Talapin, A. L. Rogach, A. Kronowski, M. Haase, and H. Weller, J. Am. Chem. Soc. 124, 11480 (2002). ${ }^{6}$ D. Weller and A. Moser, IEEE Trans. Magn. 35, 4423 (1999).

${ }^{7}$ X. Gao, Y. Cui, R. M. Levenson, L. W. K. Chung, and S. Nie, Nat. Biotechnol. 22, 969 (2004).

${ }^{8}$ S. A. Corr, Y. P. Rakovich, and Y. K. Gun'ko, Nanoscale Res. Lett. 3, 87 (2008).

${ }^{9}$ C. C. Berry and A. S. G. Curtis, J. Phys. D 36, R198 (2003).

${ }^{10}$ P. P. Freitas and H. A. Ferreira, Handbook of Magnetism and Magnetic Materials: Novel Materials, edited by H. Kronmüller and S. P. S. Parkin (New York, Wiley, 2007), Vol. 4.

${ }^{11}$ K. A. Gschneidner, V. K. Pecharsky, and A. O. Tsokol, Rep. Prog. Phys. 68, 1479 (2005).

${ }^{12}$ O. Tegus, E. Bruck, K. H. L. Buschow, and F. R. de Broer,
Nature (London) 415, 150 (2002).

${ }^{13}$ C. R. H. Bahl and K. K. Niels, J. Appl. Phys. 105, 013916 (2009).

${ }^{14}$ V. K. Tikhomirov, K. Driesen, V. Rodriguez, P. Gredin, M. Mortier, and V. V. Moshchalkov, Opt. Express 17, 11794 (2009).

${ }^{15}$ V. K. Tikhomirov, L. F. Chibotaru, D. Saurel, P. Gredin, M. Mortier, and V. V. Moshchalkov, Nano Lett. 9, 721 (2009).

${ }^{16}$ M. Mortier and G. Patriarche, Opt. Mater. 28, 1401 (2006).

${ }^{17}$ L. Néel, C. R. Acad. Sci. Paris 228, 664 (1949).

${ }^{18}$ W. F. Brown, J. Appl. Phys. 30, S130 (1959).

${ }^{19}$ V. K. Tikhomirov, D. Furniss, I. M. Reaney, M. Beggiora, M. Ferrari, M. Montagna, and R. Rolli, Appl. Phys. Lett. 81, 1937 (2002).

${ }^{20}$ D. J. M. Bevan, J. Strähle, and O. Greis, J. Solid State Chem. 44, 75 (1982).

${ }^{21}$ S. Hull, Rep. Prog. Phys. 67, 1233 (2004).

${ }^{22}$ V. K. Tikhomirov, M. Mortier, P. Gredin, G. Patriarche, C. Görller-Walrand, and V. V. Moshchalkov, Opt. Express 16, 14544 (2008).

${ }^{23}$ M. F. Hansen and S. Mørup, J. Magn. Magn. Mater. 203, 214 (1999). 
${ }^{24}$ M. F. Hanson, C. Johansson, M. S. Pedersen, and S. Mørup, J. Phys.: Condens. Matter 7, 9269 (1995).

${ }^{25}$ M. Knobel, W. C. Nunes, L. M. Socolovsky, E. De Biasi, J. M. Vargas, and J. C. Denardin, J. Nanosci. Nanotechnol. 8, 2836 (2008).

${ }^{26}$ N. W. Ashcroft and N. D. Mermin, Solid State Physics (Saunders College, Philadelphia, 1988).

${ }^{27}$ F. Wiekhorst, E. Shevchenko, H. Weller, and J. Kötzler, Phys. Rev. B 67, 224416 (2003).

${ }^{28}$ M. S. Pedersen, S. Mørup, S. Linderoth, C. Johansson, and M. Hanson, J. Phys.: Condens. Matter 9, 7173 (1997).

${ }^{29}$ V. D. Rodríguez, V. K. Tikhomirov, J. Méndez-Ramos, and A. B. Seddon, Europhys. Lett. 69, 128 (2005).

${ }^{30}$ J. S. Griffith, The Theory of Transition-Metal Ions (Cambridge University Press, London, 1971).

${ }^{31}$ K. R. Lea, M. J. M. Leask, and W. P. Wolf, J. Phys. Chem. Solids 23, 1381 (1962).
${ }^{32}$ A. Abragam and B. Bleaney, Electron Paramagnetic Resonance of Transition Ions (Clarendon Press, Oxford, 1970).

${ }^{33}$ I. B. Aizenberg, B. Z. Malkin, and A. L. Stolov, Sov. Phys. Solid State 13, 2155 (1972).

${ }^{34}$ C. G. Andeen, J. J. Fontanella, M. C. Wintersgill, P. J. Welcher, R. J. Kimble, Jr., and G. E. Matthews, Jr., J. Phys. C 14, 3557 (1981).

${ }^{35}$ R. S. Quimby, W. J. Miniscalco, and B. Thompson, J. Appl. Phys. 76, 4472 (1994).

${ }^{36}$ L. F. Chibotaru, V. K. Tikhomirov, D. Saurel, and V. V. Moshchalkov, J. Appl. Phys. 106, 053502 (2009).

${ }^{37}$ K. Baberschke, P. Pureur, A. Fert, R. Wendler, and S. Senoussi, Phys. Rev. B 29, 4999 (1984).

${ }^{38}$ L. C. Jackson, Proc. Phys. Soc. 48, 741 (1936).

${ }^{39}$ H. M. Rosenberg, Low Temperature Solid State Physics (Clarendon Press, Oxford, 1963). 